\title{
Amyloid-like Fibrils in Elastin-Related Polypeptides: Structural Characterization and Elastic Properties
}

\author{
Loretta L. del Mercato, ${ }^{* \dagger}$ Giuseppe Maruccio, ${ }^{*,}$ Pier Paolo Pompa, ${ }^{\dagger}$ Brigida Bochicchio, ${ }^{\ddagger}$ \\ Antonio M. Tamburro, ${ }^{\ddagger}$ Roberto Cingolani, ${ }^{\dagger}$ and Ross Rinaldi ${ }^{\dagger}$ \\ National Nanotechnology Laboratory of INFM-CNR, IIT Research Unit, ISUFI, University of Salento, \\ Via per Arnesano, 73100 Lecce, Italy, and Department of Chemistry, University of Basilicata, \\ Via N. Sauro, 85100 Potenza, Italy
}

Received September 11, 2007; Revised Manuscript Received November 22, 2007

\begin{abstract}
We report on the structural characterization of amyloid-like fibrils, self-assembled from synthetic polypentapeptides poly(ValGlyGlyLeuGly), whose monomeric sequence is a recurring, simple building block of elastin. This polymer adopts a $\beta$-sheet structure as revealed by circular dichroism and Fourier transform infrared spectroscopy. Furthermore, Thioflavin-T and Congo red birefringence assays confirm the presence of amyloid-like structures. To analyze the supramolecular assembly and elastic properties of the fibrils, we employed atomic force microsocopy and spectroscopy, measuring also the elasticity of mature elastin for a comparative analysis. In the case of fibrils we estimated a Young's modulus ranging from 3.5 to $7 \mathrm{MPa}$, whereas for elastin it is around $1 \mathrm{MPa}$. The possibility to section individual fibrils with nanometric control by the AFM tip, realizing biomolecular gaps in the $100 \mathrm{~nm}$ range, is also demonstrated. These results are expected to open interesting perspectives for the fabrication of protein-inspired nanostructures with specific physical and chemical properties for applications in biotechnology and tissue engineering.
\end{abstract}

\section{Introduction}

Recently, there has been great interest in fabricating novel nano(bio)materials able to self-assemble. Molecular self-assembly is ubiquitous in chemical and biological systems and consists of a spontaneous association of several individual entities into a coherent organization (like a monolayer or a nanostructure) without external instructions, through a number of specific interactions (such as covalent, electrostatic, or hydrophobic interactions), which drive self-recognition processes. In this respect, polypeptide systems are particularly attractive as building blocks with applications ranging from biosensors $^{1}$ to molecular photodiodes ${ }^{2}$ and protein transistors. ${ }^{3}$ Notably, it is possible to assemble them in complex structures, such as polypeptide nanotubes. ${ }^{4}$

A particularly well investigated example of self-assembled polypeptide structures is the amyloid fibrils, ${ }^{5,6}$ characterized by common biophysical and structural properties (including a typical X-ray diffraction pattern and a predominant $\beta$-sheet conformation), ${ }^{7,8}$ even if originated by different and apparently structurally unrelated proteins, without any primary sequence homology. In vivo, amyloids are often associated with specific diseases of different origins. ${ }^{6,9}$ On the other hand, from a technological viewpoint, amyloid fibrils have been shown to be versatile self-assembling peptide systems for applications in various fields, including molecular electronics, ${ }^{10,11}$ drug delivery, and tissue engineering. ${ }^{12-14}$

Recently, major efforts have been focused on the study of the mechanical properties of amyloids and amyloid-like fibrils with contrasting results. The main difficulties in addressing this issue lie in the complexity of the system (due to possible

* Authors to whom correspondence should be addressed. E-mail: loretta.delmercato@unile.it; giuseppe.maruccio@unile.it.

National Nanotechnology Laboratory of INFM-CNR, IIT Research Unit, ISUFI, University of Salento.

${ }^{\ddagger}$ Department of Chemistry, University of Basilicata. differences in fibril self-assembling, the arrangement of intertwined protofilaments, and the consequent structural heterogeneity) and in identifying an appropriate method to measure their elastic properties. In this respect, the atomic force microscopy (AFM) nanoindentation technique appears to be the most suitable for quantitative measurements of local elasticity, since it allows imaging of individual fibrils and then applying piconewton-nanonewton $(\mathrm{pN}-\mathrm{nN})$ forces on specific topographical features. The resulting deformations/indentations at the nanoscale can be measured by using the AFM tip as a nanoindenter. Recent reports provide values for the Young's modulus ranging from a few tens of megapascals ${ }^{4,15}$ to some gigapascals,${ }^{16,17}$ partly depending on the size of the building block $^{4,17}$ (with larger Young's modulus in nanotubes composed of small dipeptide molecules ${ }^{17}$ probably due to their closer packing density than in the case of larger proteins ${ }^{4}$ ), the experimental approach (to probe the packing density of the fibrils' $\operatorname{core}^{16}$ or the overall packing of the fibril ${ }^{15}$ ), and the applied mechanical model employed to fit the indentation data. ${ }^{15,18-20}$ Assessing and understanding the mechanical properties of amyloid-like fibrils are expected to be crucial steps toward their proposed applications for constructing mechanically resistant nanobiomaterials, such as vascular prostheses, for tissue engineering-for example as artificial fibrous scaffolds-or for drug delivery devices, if hollow. ${ }^{4}$ Moreover, these studies are expected to provide useful information about the packing density of peptides inside fibrils. As a consequence, more experiments are required to clarify the mechanical properties of fibrils.

Self-assembly into amyloid-like fibrils of elastin-related synthetic polypeptides poly(ZaaGlyGlyYaaGly) (where Zaa, Yaa $=$ Val or Leu) has been recently reported. ${ }^{21,22}$ Elastin is an insoluble extracellular matrix protein responsible for the elasticity of skin, arteries, and lungs. ${ }^{23}$ Its elastic properties and resilience depend on its supramolecular organization and association into fibers and lamellae. ${ }^{24,25}$ The gene of human 


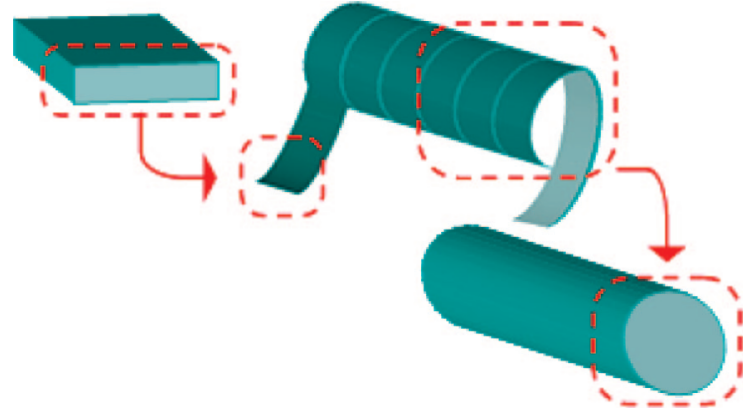

Figure 1. Self-assembly of poly(ValGlyGlyLeuGly) fibrils by twist of flat ribbon into helix/fiber. Adapted from the model proposed for poly(ValGlyGlyValGly). ${ }^{22}$

tropoelastin exhibits a cassette-like organization with distinct exons encoding for functionally and structurally distinct domains. The basic motif structure is constituted by repeating units of cross-linking domains followed by domains encoding polypeptide regions putatively responsible for the elastic properties of the protein. All of these are well characterized from a conformational point of view. ${ }^{26}$ Moreover, it has been widely demonstrated that short peptides and simple polymeric sequences comprising sequences of elastin are able to give twisted-rope filaments, with a hierarchical supramolecular organization similar to that found for the entire protein. ${ }^{27-31}$

Here we report on the self-assembly and structural characterization of synthetic poly(ValGlyGlyLeuGly) polypentapeptide amyloid-like fibrils by a variety of techniques. Circular dichroism (CD) and Fourier transform infrared (FTIR) spectroscopy were used to probe the protein conformation, while Congo red birefringence and Thioflavine-T staining assay provided evidence for the formation of amyloid fibrils. Then we employed AFM and spectroscopy to analyze their supramolecular structure and elastic properties. The possibility to section individual fibrils with nanometric control using the AFM tip is also demonstrated, with possible applications in the field of molecular electronics. ${ }^{10,11}$ The sequence chosen for the monomer (ValGlyGlyLeuGly) is highly repeated in elastin. ${ }^{32}$ Elastin-inspired biopolymers have been found of large interest in recent years ${ }^{33,34}$ because they may be suitable for the development of novel biomaterials. Studies on their sequence-structure relationship can be important for the design of prosthetic biomaterials with tuned elastic/ mechanical properties ${ }^{35}$ for biomedical applications (e.g., tissueengineered skin grafts). As a reference compound, we studied samples of native, cross-linked elastin.

\section{Experimental Section}

Sample Preparation. Poly(ValGlyGlyLeuGly) (specific viscosity $[\eta]=0.20 \mathrm{~g} \cdot \mathrm{mL}^{-1} \mathrm{c}, 1 \%$ dichloroacetic acid) was chemically synthesized following the procedures described in Tamburro et al. ${ }^{32}$ Accordingly to the values found for analogous sequences, this intrinsic viscosity corresponds to $5000<M_{\mathrm{w}}<10000\left(M_{\mathrm{w}}\right.$, weight-average molecular weight). The polydispersity was not determined but a lognorm type distribution with large polydispersity is tipically found for polypeptides obtained by polycondensation reactions.

Poly(ValGlyGlyLeuGly) fibrils were generated by suspending the dry powder peptide at a concentration of $1.0 \mathrm{mg} \cdot \mathrm{mL}^{-1}$ in ultrapure water (10 M $\Omega \mathrm{cm}$, Elga Water) and incubating the solution in a vial tube for several (typically 3-5) weeks at $27^{\circ} \mathrm{C}$ in order to obtain mature amyloid-like fibrils. ${ }^{21}$ According to the general model recently proposed for poly(ValGlyGlyValGly), ${ }^{22}$ the self-assembly of the polypeptide in water proceeds through a multipathway assembling scheme, which involves the twist of a flat ribbon into a helix/fiber (Figure 1).
Native cross-linked elastin from bovine neck ligament (insoluble, lyophilized powder) was purchased from Sigma (St. Louis, MO). Native elastin samples were prepared by suspending the lyophilized powder at a concentration of $1.0 \mathrm{mg} \cdot \mathrm{mL}^{-1}$ in ultrapure water $(10 \mathrm{M} \Omega \mathrm{cm}$, Elga Water) and incubating the solution in a vial tube for 3 months at $27{ }^{\circ} \mathrm{C}$.

CD Spectroscopy. CD spectra for the peptides were obtained using a Jasco J-600 spectropolarimeter at various temperatures and at concentrations of $0.25 \mathrm{mM}$ in $70 \%$ TFE and $10 \%$ HFIP by using cells of $0.1 \mathrm{~cm}$. These conditions were needed because the polymer is fully insoluble in water but soluble in fluorinated alcohols. Different percentages were chosen because they correspond to the maximum of water content allowing the solubility of the polymer in the two different mixtures. Spectra were acquired in the range $190-250 \mathrm{~nm}$ at a temperature of 0,25 , and $60{ }^{\circ} \mathrm{C}$ by taking points every $0.1 \mathrm{~nm}$, with $20 \mathrm{~nm} \cdot \mathrm{min}^{-1}$ scan rate, an integration time of $2 \mathrm{~s}$, and a $1 \mathrm{~nm}$ bandwidth. The data are expressed in terms of $[\Theta]$, the molar (per pentameric unit) ellipticity in units of degree $\mathrm{cm}^{2} \cdot \mathrm{dmol}^{-1}$.

FTIR Spectroscopy. The polypeptide was analyzed by FTIR in $\mathrm{KBr}$ pellets in the dry powered form. The spectra were recorded on a Jasco spectrometer and then smoothed by using the Savitzky-Goolay algorithm. The samples were examined at the solid state in $\mathrm{KBr}$ pellets $(1 \mathrm{mg} / 100 \mathrm{mg}$ ). The decomposition of FTIR spectra was obtained using GRAMS32 software, and the number of peaks in the FTIR spectra decomposition has been fixed as the minimum possible. The percentage of Gaussian and Lorentzian functions was fixed at the 8:2 ratio.

Congo Red Birefringence Assay. The staining solution was prepared by using $80 \% \mathrm{EtOH}, 20 \%$ of ultrapure water $(10 \mathrm{M} \Omega \mathrm{cm}$, Elga Water) and a saturating amount of $\mathrm{NaCl}$. The solution was stirred for a few minutes, and the excess $\mathrm{NaCl}$ was filtered away. Then a saturating amount of Congo red was added and the mixture was stirred and filtered to obtain the final working solution. ${ }^{36}$ Poly(ValGlyGlyLeuGly) was almost dissolved in a mixture of $10 \%$ HFIP and $90 \%$ water, then, after centrifugation, the supernatant was kept at $60{ }^{\circ} \mathrm{C}$ for $48 \mathrm{~h}$. Fibers were observed at the bottom of the test tube. Fifty microliters of solution was deposited onto a glass slide and, after airdrying, Congo red was added. The excess solution was blotted away, and the sample was allowed to dry at room temperature. ${ }^{37}$ The stained sample was examined under polarized light with a Nikon ECLIPSE E600 POL microscope equipped with Nikon Cool Pix 4500 4.0 Mega Pixel.

Th-T Imaging Experiments by Confocal Microscopy. The fluorophore thioflavin-T (Th-T) (Sigma) was used for the assessment of amyloid structures in the self-assembled fibrils from the synthetic polypentapeptides poly(ValGlyGlyLeuGly). Fibril samples were prepared by cast deposition of a $5 \mu \mathrm{L}$ drop of fibril suspension (1.0 $\mathrm{mg} \cdot \mathrm{mL}^{-1}$ in ultrapure water) onto a silicon dioxide $\left(\mathrm{SiO}_{2}\right)$ substrate. The samples were then stained with thioflavin- $\mathrm{T}$ (5 $\mu \mathrm{M}$ aqueous solution, 5 min incubation time) and kindly washed with deionized water before imaging. Spatially resolved fluorescence images of the thioflavin-T stained fibrils were taken by a confocal microscope (Leica TCS-SP5). Th-T dye was excited at $488 \mathrm{~nm}$, and emission was collected in the 510-600 nm range, through a $63 \times, 1.40 \mathrm{NA}$ oil immersion objective.

AFM Measurements. AFM measurements were performed in air, at ambient conditions $\left(20-25{ }^{\circ} \mathrm{C}\right.$, atmospheric pressure, $\sim 50 \%$ of humidity), using a CP-II scanning probe microscope (Veeco, SantaBarbara, CA) equipped with 5 or $100 \mu \mathrm{m}$ scanners. Silicon probes (MPP-11100, Veeco Probes) with a nominal spring constant of 40N/m and a resonance frequency of $300 \mathrm{kHz}$ were used for AFM imaging in tapping mode. For both force-vs-distance $(F-d)$ experiments and mechanical tests, we employed silicon nitride probes (DNP-S, Veeco Probes) whose spring constant was measured by the thermal tune method (only AFM cantilevers having shifts smaller than 5\% from the $0.58 \mathrm{~N} / \mathrm{m}$ nominal values provided by the manufacturer were used in order to have similar tips in different indentation experiments). 
Fibril samples were prepared by cast deposition of a $20 \mu \mathrm{L}$ drop of

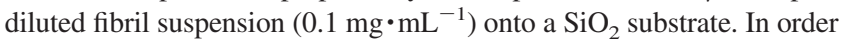
to avoid any fibril deformation during contact mode imaging, a low set point of a few tens of piconewton and a scan rate of $0.5-1 \mathrm{~Hz}$ were used. Elastin samples were prepared by means of the same procedure used with poly(ValGlyGlyLeuGly) fibrils (drop casting).

$F-d$ curves were acquired by collecting some ten force curves at different locations, on the top of individual poly(ValGlyGlyLeuGly) fibrils, checking for their reproducibility. The deflection sensitivity of the instrument was calibrated for each experiment by performing several $F-d$ curves directly on the $\mathrm{SiO}_{2}$ substrate. After force measurements were completed, another topographic image at the same position was carried out to confirm that (i) the sample's drift was negligible during the period of force measurements and (ii) the applied stresses were well within the elastic regime of the structures and did not lead to damage of the fibrils (i.e., permanent deformation at the indentation points).

\section{Results and Discussion}

Circular Dichroism Spectroscopy. Figure 2a shows the CD spectra of poly(ValGlyGlyLeuGly) in 70\% TFE at different temperatures. Only slight effects induced by the temperature are appreciable except at $25^{\circ} \mathrm{C}$ where a strong decrease of the positive band is observed. Figure $2 \mathrm{~b}$ shows the $\mathrm{CD}$ spectra of poly(ValGlyGlyLeuGly) in 10\% HFIP at the indicated temperatures. At $0{ }^{\circ} \mathrm{C}$ the spectrum is characterized by a prominent negative band at about $217 \mathrm{~nm}$ and by a positive one below $200 \mathrm{~nm}$. This spectrum is diagnostic of a $\beta$-strand conformation and is only slightly changed on increasing the temperature: the negative band is at a first stage reduced at $25^{\circ} \mathrm{C}$ and then slightly increased at $60{ }^{\circ} \mathrm{C}$. These weak effects could be attributed to $\beta$-strands with increased flexibility between 0 and $25{ }^{\circ} \mathrm{C}$ followed by a cross- $\beta$ sheet structure at $60^{\circ} \mathrm{C}$. This structure is indeed favored by a nonexcessive increase of temperature. The role of the aqueous component is easily appreciated when the spectrum of the polymer at room temperature in either pure HFIP or TFE is taken into account (Figure 2c): here the negative band at around $200 \mathrm{~nm}$, essentially due to extended conformations, is dominant.

FTIR Spectroscopy. The deconvoluted FTIR spectra of the amide I, II, and A regions of the polypeptide are shown in parts $\mathrm{a}, \mathrm{b}$, and c of Figure 3, respectively. The amide I region contains three main components at 1678 , at 1653 , and at $1631 \mathrm{~cm}^{-1}$ suggesting a dominance of beta conformations together with unordered components.

In fact, the first and the third components are indicative of antiparallel $\beta$-sheet and cross- $\beta$ structures, respectively, ${ }^{38-40}$ while we assign the component at $1653 \mathrm{~cm}^{-1}$ to unordered conformations since the $\alpha$-helical structure is very unlikely for a sequence such as that of poly(ValGlyGlyLeuGly).

This interpretation is supported by the observed band at 1531 $\mathrm{cm}^{-1}$ in the amide II region (Figure 3b), which is normally attributed to the dominance of the bending mode of hydrogen bonded $\mathrm{NH}$ groups in antiparallel $\beta$-sheet structure. ${ }^{41}$ Additionally, the minor component at $1556 \mathrm{~cm}^{-1}$ could also be assigned to $\beta$-sheet conformation, ${ }^{41,42}$ while we ascribe the band at 1575 $\mathrm{cm}^{-1}$ to free $\mathrm{NH}$ groups.

Finally, the amide A region shows a prominent band at 3287 $\mathrm{cm}^{-1}$ attributable to the stretching mode of hydrogen-bonded $\mathrm{NH}$ groups. The band at $3385 \mathrm{~cm}^{-1}$ is most probably due to free $\mathrm{NH}$ groups. In addition, the amide A region of poly(ValGlyGlyLeuGly) shows also the presence of significant absorptions originating from the $\mathrm{OH}$ stretching of water at 3432 and at $3512 \mathrm{~cm}^{-1}$.
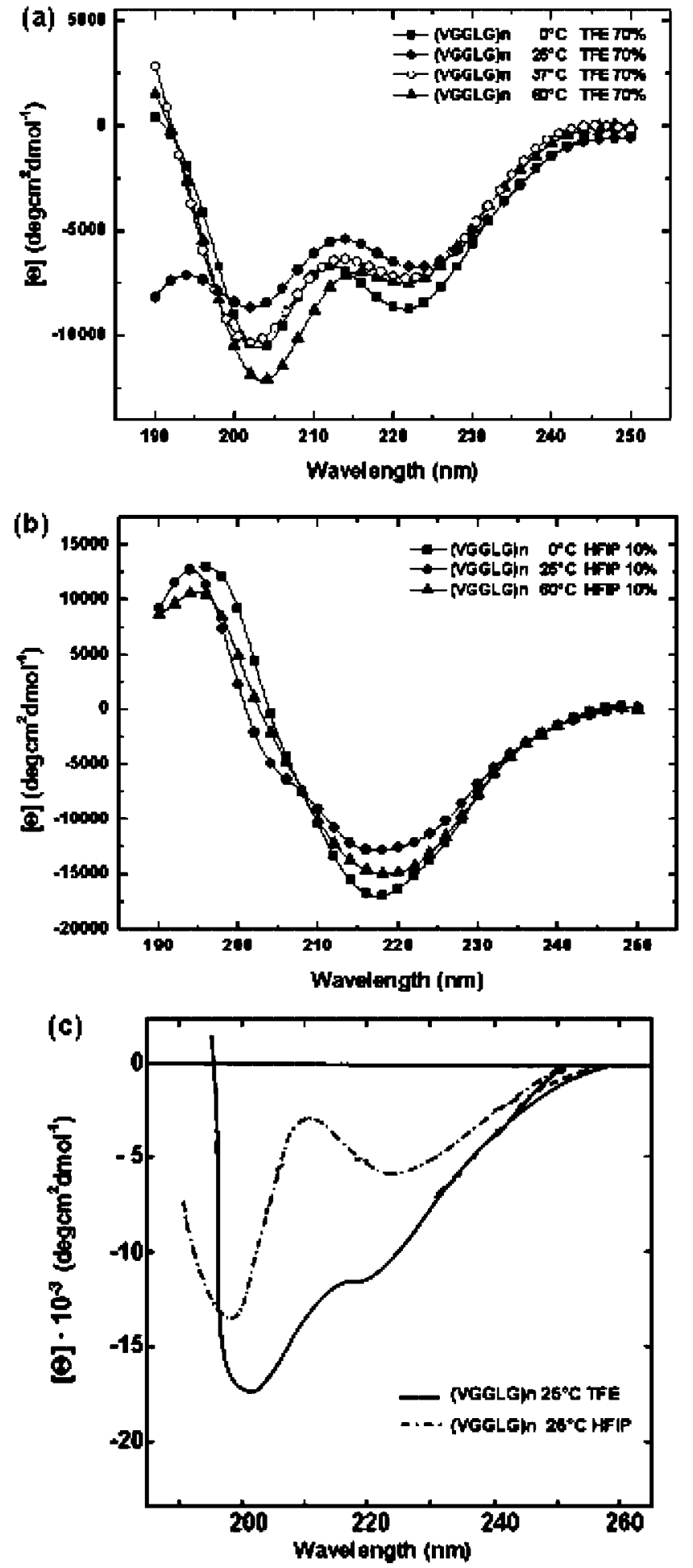

Figure 2. (a) CD spectra of poly(ValGlyGlyLeuGly) in $70 \%$ TFE: 0 ${ }^{\circ} \mathrm{C}(\boldsymbol{\square}), 25^{\circ} \mathrm{C}(\boldsymbol{\bullet}), 37^{\circ} \mathrm{C}(\mathrm{O}), 60^{\circ} \mathrm{C}(\mathbf{\Delta})$. (b) $\mathrm{CD}$ spectra of poly(ValGlyGlyLeuGly) in $10 \%$ HFIP: $0^{\circ} \mathrm{C}(\boldsymbol{\square}), 25^{\circ} \mathrm{C}(\boldsymbol{\bullet}), 60^{\circ} \mathrm{C}(\mathbf{\Delta})$.

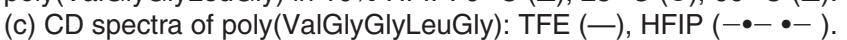
(Reproduced with permission from ref 32. Copyright 1992 Adenine Press.)

Congo Red Birefringence and Th-T Staining Assay. Congo red birefringence and Th-T binding assay permit characterization of the presence of amyloid fibrils and also their rates of formation in vitro. ${ }^{43-45}$

When Congo red was added to the fibril-forming polypeptide solutions, visible red fibers were observed at the bottom of the test tube. To view the fibrils under a light microscope, the 

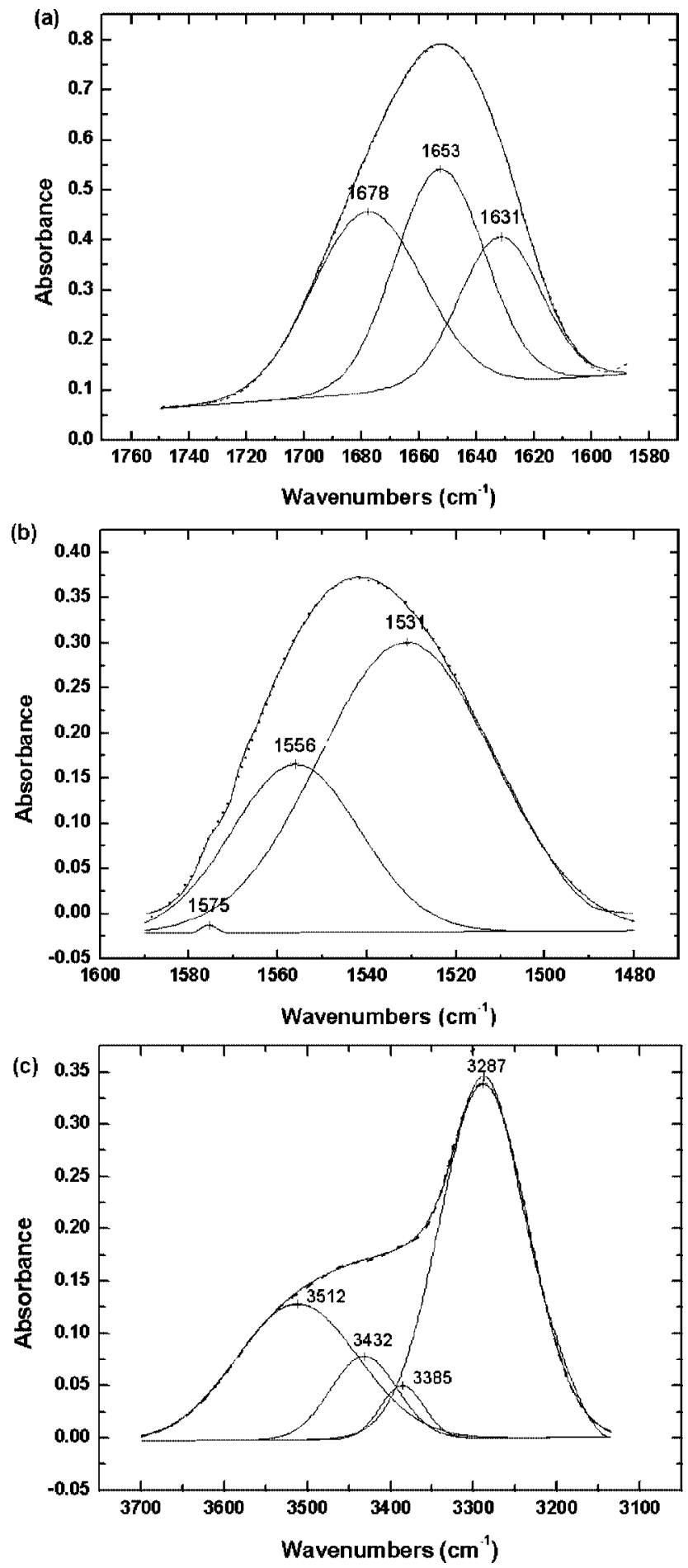

Figure 3. FTIR deconvolution spectrum: (a) amide I, (b) amide II, and (c) amide A regions of poly(ValGlyGlyLeuGly) in $\mathrm{KBr}$ pellet; experimental curve (solid line), fitted curve (dotted line).

sample solution was deposited onto a glass slide and, after airdrying, Congo red was added. As a result, the fibrils were found to coalesce giving rise to an object that, when viewed under cross-polarized light, exhibited the characteristic birefringence going from red to yellow to green (Figure 4a,b). This behavior is typical of amyloids (see for example ref 46).

To investigate further the presence of amyloid-like structures in poly(ValGlyGlyLeuGly) fibrils, we carried out Th-T staining assay. Direct binding to amyloid-like fibrils in the solid state with the fluorochrome Th-T was assessed by confocal microscopy imaging. Figure 4c,d shows two representative fluorescence images of the Th-T stained fibrillar nanostructures $\left(\lambda_{\text {exc }}\right.$ was $488 \mathrm{~nm}$ ), revealing the typical structural features of the fibrils, similar to those observed by AFM (see below). In some regions, single bright mature fibrils were also detectable (Figure 4d).

AFM Studies. Fibril samples for AFM studies were prepared by cast deposition of a $20 \mu \mathrm{L}$ drop of diluted fibril suspension $\left(0.1 \mathrm{mg} \cdot \mathrm{mL}^{-1}\right)$ onto a $\mathrm{SiO}_{2}$ substrate (see Experimental Section). After solvent evaporation, the protein structures were characterized by AFM in tapping mode and/or in contact mode at ambient conditions. In Figure 5, typical AFM images of poly(ValGlyGlyLeuGly) fibrils at different spatial resolution are shown. We observed amyloid-like fibrils with different sizes and a significant degree of structural heterogeneity which is likely to reflect the different arrangement of intertwined protofilaments who are the constituents of the fibrils. Globular particles-peptide aggregates/oligomers-distributed over the entire surface among the fibrillar structures were also detected (Figure 5a). According to Jansen et al., ${ }^{47}$ these aggregates at a preamyloidal state act as nucleation centers and their oligomerization leads to the formation of the protofilaments which merge and intertwine, yielding thin fibrils capable of further association to produce mature amyloids. ${ }^{4,32}$ Hydrophobic interactions are postulated to be the driving force which leads polypeptide molecules to form fibrils (with protofibrils and protofilaments as intermediate steps) and participate in the lateral aggregation of monomers/oligomers and fibrils. Thus, the formation of mature amyloid-like fibrils can be likely ascribed to a process aimed to minimize the exposure of hydrophobic residues. ${ }^{47}$

In some regions of the same sample, especially near the edge of the deposited drop, we mainly observed bundles of bended fibrils randomly lying on the silicon dioxide substrate with little tendency to criss-cross and with lengths longer than $1 \mu \mathrm{m}$ (Figure 5b). The measured diameters (at full width half-maxima) and heights range from 100 to $300 \mathrm{~nm}$ and from 10 to $50 \mathrm{~nm}$, respectively. Taking into account the tip convolution, from the apparent lateral dimension, we estimated the real fibril width to be in the range $60-250 \mathrm{~nm}$ (with a peak in the distribution around $150 \mathrm{~nm}$ ). Figure 5c,d shows poly(ValGlyGlyLeuGly) fibrils with a characteristic domain texture. Each fibril consists of several filaments arranged in a roughly twisted pattern, presumably originating by side-by-side interaction. In Figure 5e, we report a line profile for the fibril shown in Figure 5d, characterized by a height of $17 \mathrm{~nm}$ and an apparent lateral dimension of $210 \mathrm{~nm}$ (corresponding to approximately $170 \mathrm{~nm}$ after tip deconvolution). A high-resolution three-dimensional (3D) image of an individual fibril is reported in Figure 5f. The AFM image shows a clear, periodic pattern on the fibril surface, which can be attributed to protofilaments wound around each other, consistent with commonly accepted structural models for amyloid fibrils. ${ }^{48}$ In general, the heterogeneity of fibrils results in filament pitches (axial rises per filament turn) ranging from 350 to $700 \mathrm{~nm}$ and filament spacing from 35 to $350 \mathrm{~nm}$, corresponding to 2-start/10-start filaments. Moreover, a righthanded filament twist is clearly visible. We found both rightand left-handed helical and filament orientation in the selfassembled polymer structures (likely due to the significant structural heterogeneity of the fibrils). This finding is consistent with a recent study carried out on poly(ValGlyGlyValGly). ${ }^{22}$

Mechanical Properties. To determine the local elasticity of individual fibrils, we employed a nanoindentation technique to apply forces in the nanonewton range and to measure the resulting indentations at the nanoscale. The experimental setup for the indentation measurements is sketched in the inset of Figure 6a. In particular, we focused on fibrils having lateral 

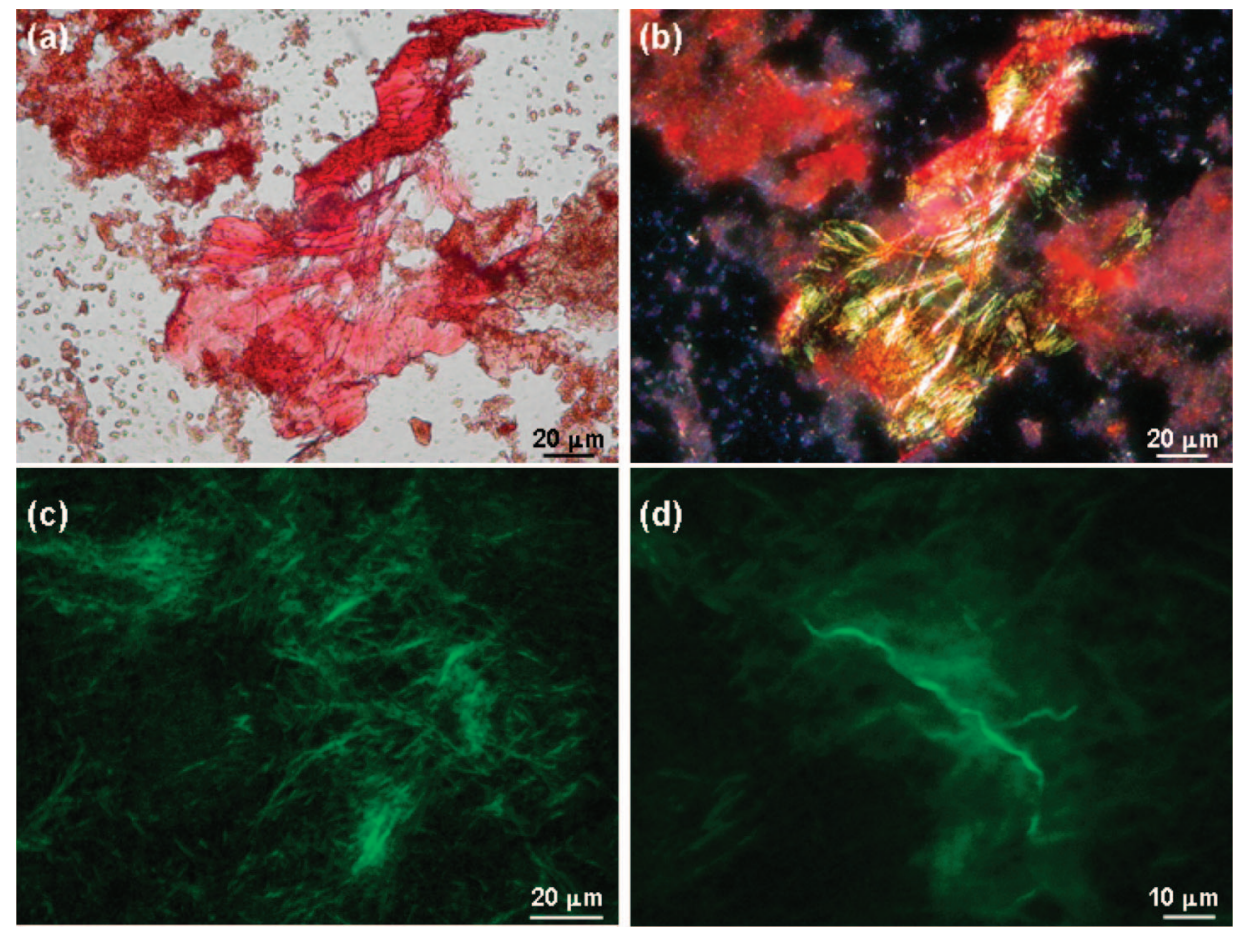

Figure 4. (a, b) Optical microscopy images of poly(ValGlyGlyLeuGly) fibrils stained with Congo red: (a) bright field and (b) polarized light. (c, d) Two representative confocal microscopy images of the Th-T stained fibrillar structures. The excitation wavelength was $488 \mathrm{~nm}$, and the Th- $T$ emission was collected in the $510-600 \mathrm{~nm}$ range. ${ }^{44,45}$
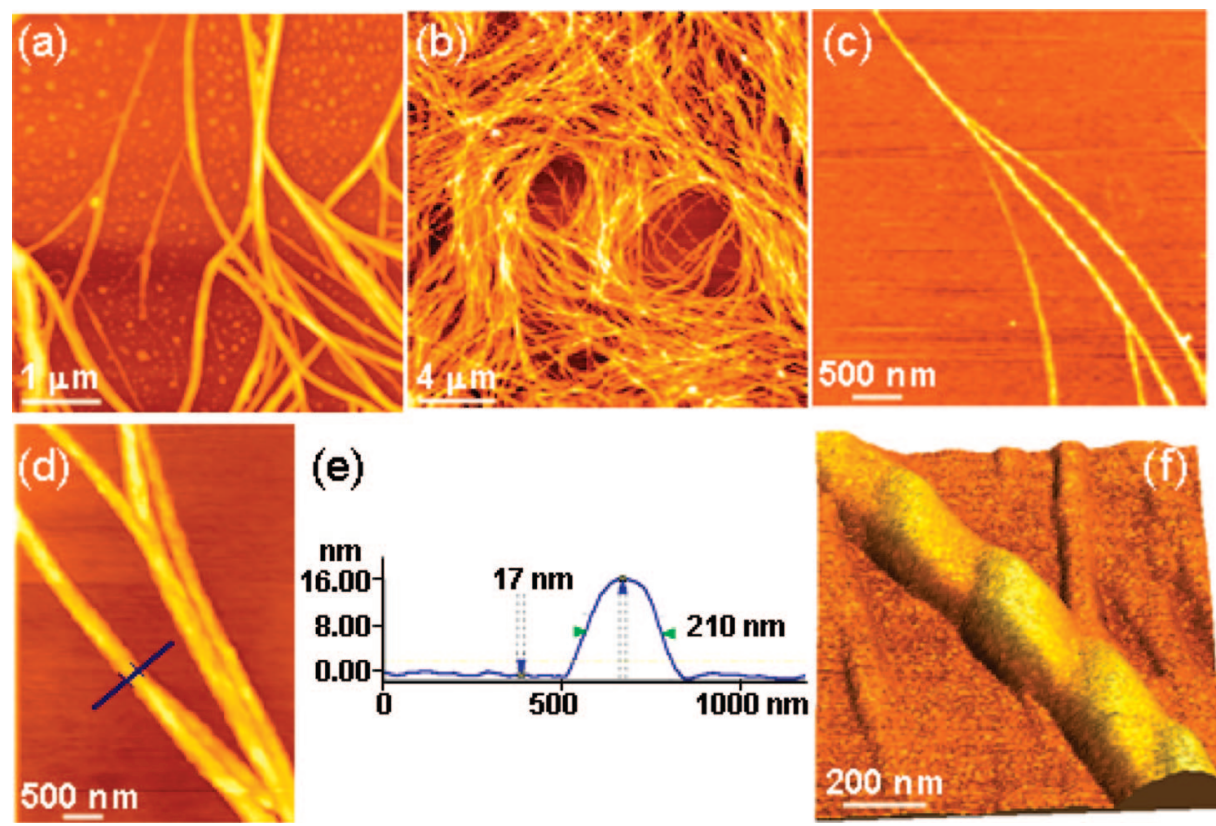

\section{(e)}
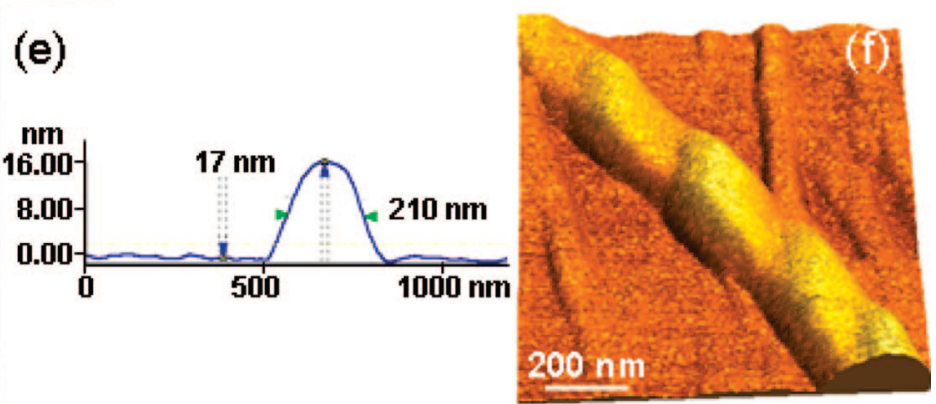

Figure 5. AFM characterization of poly(ValGlyGlyLeuGly) amyloid-like fibrils. (a) Globular particles (peptide aggregates/oligomers) are visible among the fibrillar structures, while in some regions of the sample (b) bundles of bended fibrils are detectable (see the text). (c, d) Two representative AFM images of isolated and almost straight fibrils. (e) Line profile from the fibril shown in panel d, characterized by a height of $17 \mathrm{~nm}$ and an apparent lateral dimension of $210 \mathrm{~nm}$ (corresponding to approximately $55 \mathrm{~nm}$ after tip deconvolution). (f) High-resolution 3D image of an individual poly(ValGlyGlyLeuGly) fibril. The clear, periodic pattern on the fibril surface can be attributed to protofilaments wound around each other.

dimensions in the range 150-200 $\mathrm{nm}$ (the most represented on the samples). To convert the raw cantilever deflection vs probe displacement data into force-distance relations, we calculated the deflection sensitivity by performing a measurement on a hard substrate $\left(\mathrm{SiO}_{2}\right)$ and used the tip measured spring constants. ${ }^{35}$

In Figure 6a, $F-d$ curves acquired on the hard substrate (cantilever deflection) and three representative fibrils are compared. As the cantilever approaches the surface (in the noncontact region), no deflection is measured, indicating the absence of long-range attractive/repulsive forces. After jumping into contact, if the cantilever is sufficiently stiff, the probe tip indents into the surface providing information about the local elasticity. We found the force to increase linearly with distance up to an indentation of approximately $10-15 \mathrm{~nm}$, with occasional changes in the slope. No detectable hysteresis was observed at the scan speed investigated $(1000 \mathrm{~nm} / \mathrm{s})$, meaning a purely elastic response of the fibrils with a negligible viscous compo- 


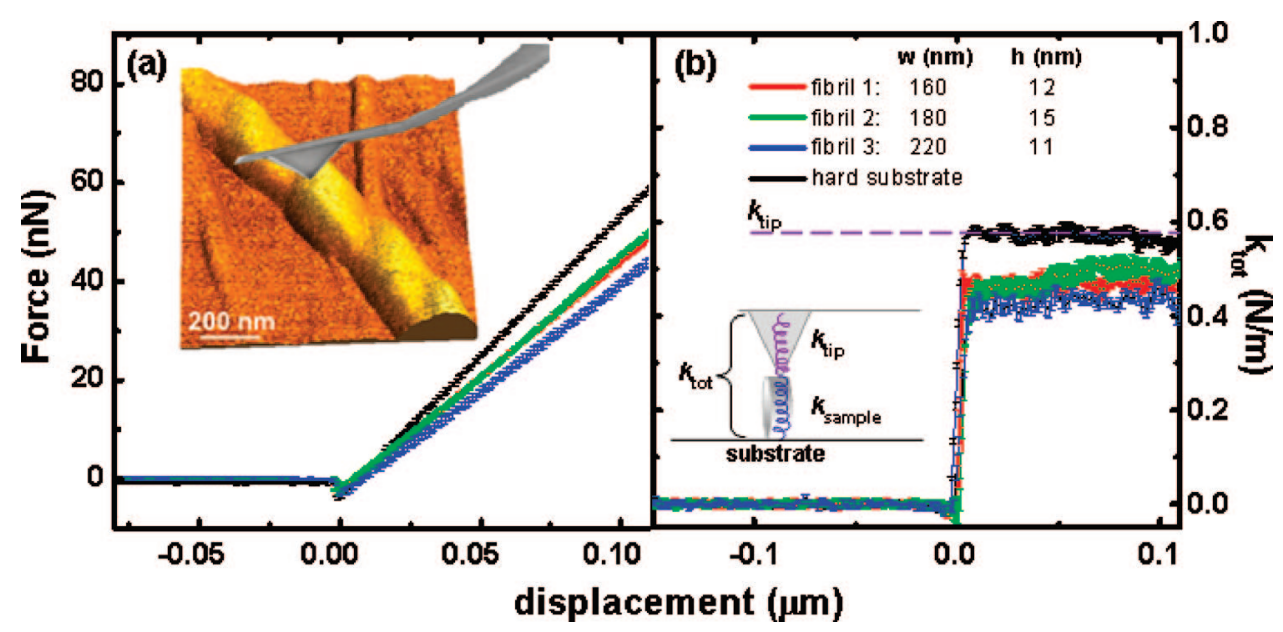

Figure 6. (a) Force-displacement curves and scheme of the setup for the AFM nanoindentation experiments (Inset a). (b) Measured total contact stiffness $\left(k_{\text {tot }}\right)$ for the hard substrate and three representative fibrils (w, width; $h$, height). The (small) statistical errors evaluated from different measurements are reported as error bars. From the $k_{\text {tot }}$ values shown, assuming the fibril and the cantilever act as two springs in a series (see Inset b), we calculated the fibril stiffness $\left(2.28,2.28\right.$, and $1.53 \mathrm{~N} / \mathrm{m}$ for fibrils $1-3$, respectively). If $k_{\text {sample }}$ is much higher than $k_{\text {tip }}$, $k_{\text {tot }}$ will be close to $k_{\text {tip }}$. Dashed line represents cantilever stiffness of the employed tip $\left(k_{\text {tip }}=\right.$ around $\left.0.58 \mathrm{~N} / \mathrm{m}\right)$.

nent ${ }^{4}$ in the investigated range. The total contact stiffness $k$ can be extrapolated from the slope of these curves and is shown in Figure $6 \mathrm{~b}$ for three fibrils and for the hard substrate (in this last case it corresponds to the cantilever stiffness). In particular, we report data from three different fibrils whose stiffness values cover the full observed range (from minimum to maximum) and their widths/heights are 160/12 nm, 180/15 nm, and 220/ $11 \mathrm{~nm}$, respectively. The fibril stiffnesses can be calculated from the measured total values taking into account the cantilever stiffness ( $k_{\text {tip }}=$ around $0.58 \mathrm{~N} / \mathrm{m}$ ), according to the following relation

$$
k_{\mathrm{s}}=\frac{k_{\mathrm{tip}} k_{\mathrm{tot}}}{k_{\mathrm{tip}}-k_{\mathrm{tot}}}
$$

where we assume the fibril and the cantilever to act as two springs in series (see inset of Figure 6b). We obtained 2.28, 2.28 , and $1.53 \mathrm{~N} / \mathrm{m}$ for fibrils 1,2 , and 3, respectively. It is interesting to note that fibrils 1 and 2 exhibit similar aspect ratio and stiffness. This could suggest similar packing densities of peptides (but different from fibril 3).

To estimate the Young's modulus of the fibrils, we used the same approach of Guo et al. ${ }^{15}$ From the Hertzian model ${ }^{49}$

$$
F^{23}=C^{*}-\left[\frac{4}{3} \frac{E R^{1 / 2}}{\left(1-\sigma^{2}\right)}\right]^{2 / 3} \Delta
$$

where $\Delta$ is the tip-sample distance (related to the indentation $\delta$ with some constant $C: \delta=C-\Delta$ ), $F$ is the load, $R$ is the radius of curvature of the probe, $E$ is the Young's modulus, and $\sigma$ is Poisson's ratio of the material, while $C^{*}$ is another constant. Thus, Young's modulus can be estimated from the slope of $F^{2 / 3}$ versus distance. Using a Poisson ratio value $\sigma$ around 0.3 as a reasonable guess ${ }^{15}$ for fibrils, we obtain 30,30 , and $18 \mathrm{MPa}$ for fibrils $1-3$, respectively. However, since a soft fibril lying on a hard substrate was indented and the radius of the contact area is comparable to the sample thickness, the influence of the underlying substrate on the indentation has to be taken into account to evaluate Young's modulus with greater accuracy. As a result, it is necessary to consider a correction factor, depending on the maximum applied load, the sample thickness, the Poisson's ratio, Young's modulus, and the tip radius. ${ }^{15,19,50,51}$ According to the model used by Guo et al., ${ }^{15}$ we calculated correction factors in the range of 4-5 for the three fibrils. As a consequence, the corrected values of Young's modulus are 5.8, 7.1, and 3.7 MPa for fibrils $1-3$, respectively (using the Guo model to calculate the correction factor, we lost the correlation with the fibril aspect ratio and further experiments are in progress in our laboratories to better understand this issue).

As a reference, we also measured Young's modulus of crosslinked mature elastin. Elastin samples for AFM studies were prepared by the same procedure used for poly(ValGlyGlyLeuGly) polypeptides (i.e., drop casting) in order to have a direct comparison of the microstructural and elastic properties of both native bovine elastin and un-cross-linked poly(ValGlyGlyLeuGly) samples. In the case of elastin, we observed globular structures randomly distributed on the surface (see Figure S1 in Supporting Information). We thus carried out indentation experiments on the top of these globular features, obtaining an uncorrected Young's modulus of 3.3 MPa, which becomes 1.1 MPa after correction. Previous determinations of Young's modulus of elastin by means of macroscopic techniques $^{52}$ gave similar results (see Table 1, where Young's modulus for fibrils and elastin from different reports are summarized). However, to the best of our knowledge, this is the first time that the elastic properties of both native elastin and a synthetic elastin-like polypeptide have been measured with the same technique in a comparative study. As a consequence, we believe these results validate our procedure for the determination of Young's modulus. Since variations exceed the random error of the measurements (Figure 6a,b), the measured distribution of elasticity among fibrils can be tentatively ascribed to their intrinsic structural heterogeneity observed in AFM images, whereas the difference in Young's modulus between them and the parental protein elastin might be attributed to a different packing density of peptides (although the difference in their molecular weight and their structural conformation might also play a role). From this point of view, it is of particular interest that Young's moduli of un-cross-linked poly(ValGlyGlyLeuGly) and cross-linked bovine elastin are in the same order of magnitude. Within this context are our current studies on cross-linked poly(OrnGlyGlyOrnGly), ${ }^{53}$ where the ornithine residues were chosen just for obtaining functional groups to be cross-linked by glutaric aldehyde.

To further investigate the mechanical resistance of single fibrils and their applicability in nanobiotechnology, we per- 
Table 1. Comparison of the Elastic Modulus of Amyloid/Amyloid-like Fibrils and Elastin Reported in the Literature

\begin{tabular}{llcc}
\hline ref & \multicolumn{1}{c}{ protein type } & elastic modulus (MPa) & method \\
\hline 17 & Phe-Phe dipeptide amyloid fibrils & 19000 & AFM nanoindentation \\
16 & insulin amyloid fibrils & $2900-3700$ & AFM nanoindentation \\
15 & insulin amyloid fibrils & $5-50$ & AFM nanoindentation \\
this work & poly(ValGlyGlyLeuGly) amyloid-like fibrils & $3-7$ & AFM nanoindentation \\
this work & native elastin & 1.1 & AFM nanoindentation \\
52 & native elastin & 1.1 & macroscopic techniques \\
58 & native elastin & $0.1-1.2$ & macroscopic techniques \\
59 & native elastin & $0.3-0.6$ & macroscopic techniques \\
\hline
\end{tabular}
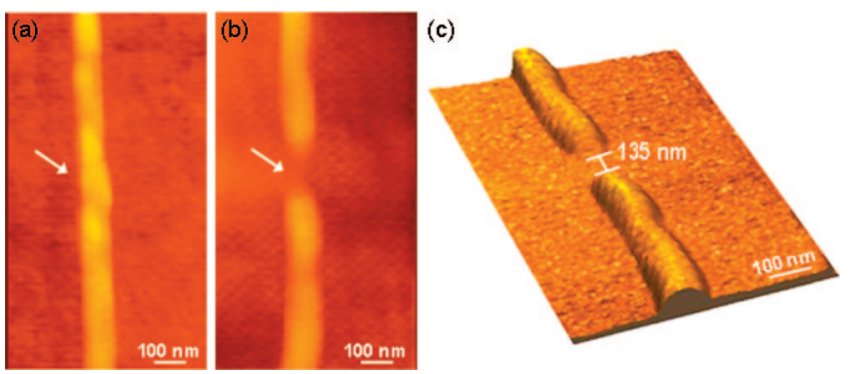

Figure 7. AFM images acquired at low setpoints (a few tens of piconewtons), in order to avoid fibril damage, of a straight fibril before (a) and after (b) the AFM manipulation (nanosectioning). The fibril was cut by increasing the contact mode setpoint to around $50 \mathrm{nN}$ in the scan lines indicated by the arrow. (c) Three-dimensional image after fracture. A final gap of $135 \mathrm{~nm}$ was obtained. The samples were scanned in contact mode at ambient conditions $\left(20-25^{\circ} \mathrm{C}\right.$, atmospheric pressure, $\sim 50 \%$ of humidity).

formed AFM nanosectioning experiments to locally fracture single fibrils and monitored the changes of morphology. Typically, we acquired AFM images on straight fibrils at low setpoints (a few tens of piconewtons) in order to avoid fibril damage (Figure 7a). To achieve manipulation and perform direct mechanical tests at a specific position (denoted by an arrow in Figure 7), we temporarily increased the contact-mode setpoint to around $50 \mathrm{nN}$ in the corresponding scan lines while collecting the subsequent images. As a result, we observed a partial cut (a nanometer-sized gap) in the fibril when we acquired a last image (Figure 7b) at low setpoint (a few tens of piconewtons). Figure 7c shows a three-dimensional AFM image of the fibril after fracture. A longitudinal cross section of the fibril reveals a gap of $135 \mathrm{~nm}$. We also observed an apparent correlation between the efficiency of AFM manipulation and the morphology of the fibrils, with a special influence of the fibril diameter that we can tentatively ascribe to internal structural heterogeneity (due to a different wall thickness or packing density). Experiments aimed at investigating in more detail the relationship between fibril's diameter and mechanical resistance are in progress.

\section{Conclusions}

In conclusion, we have investigated amyloid-like poly(ValGlyGlyLeuGly) fibrils by a variety of techniques. CD and FTIR spectroscopy measurements, together with Congo red birefringence and Thioflavin-T staining assays, have shown the ability of poly(ValGlyGlyLeuGly) polypeptides to adopt a cross- $\beta$ structure that favors the assembly into amyloid-like fibrils. Morphology and elastic properties of these fibrillar materials have been analyzed by means of AFM and nanoindentation experiments. As a reference, we also measured the elastic modulus of the parental protein elastin. The possibility to selectively cut single fibrils by the AFM tip and realize biomolecular gaps in the $100 \mathrm{~nm}$ range has been also reported.
This may lead to applications in molecular electronics, for example for the fabrication of nanoscaled junctions by metallization. ${ }^{10,11}$ The peculiar features of these peptide nanostructures, along with the possibility to specifically engineer their amino acid sequence (in order to obtain smart materials with unique chemical and biological features), are expected to result in significant advantages for biomolecular electronics and tissue engineering applications. Experiments to bioengineer the polypeptide sequence for specific applications are currently underway in our laboratories.

Finally, the results reported in the present paper are important also within the framework of biological (and pathological) function of elastin. As a matter of fact, in a recent paper ${ }^{54}$ some of us have proposed a biological role for amyloidogenic sequences of elastin. These sequences, of the general type ZaaGlyGlyYaaGly (Zaa, Yaa = Val or Leu), are found in peptides excised from the parent protein by enzymatic attack of elastases. Accordingly, one can hypothesize that under pathological conditions, such as high lipid concentration, the enzymes can attack more quickly elastin, ${ }^{55}$ giving rise to amyloidogenic sequences that are able to deposit fibers favored by the lipids themselves. ${ }^{56}$ If confirmed by aggregation studies of such amyloid-like fibrils under physiological conditions, this mechanism might explain the so-called "elastotic material" found in the arteries of patients affected by vasculopathies. ${ }^{57}$

Acknowledgment. The authors gratefully acknowledge the support by the Italian Ministry of Research ("FIRB" project: RBLA03ER38_001) and by the EU (Elastage Contract No. 018960). Moreover, we thank Franco Calabi, Stefania Sabella, and Valentina Arima for fruitful discussions and Eliana D'Amone for technical assistance.

\section{Nomenclature}

AFM: atomic force microscopy

CD: circular dichroism

FTIR: Fourier transform infrared

TFE: trifluoroethanol

HFIP: hexafluoroisopropanol

Th-T: thioflavin-T

Note Added after ASAP Publication. This article was released ASAP on February 8, 2008. The e-mail address for Giuseppe Maruccio and Figure 6 have been revised. The corrected version posted on February 13, 2008.

Supporting Information Available. Typical topographic images of cross-linked bovine elastin samples deposited onto $\mathrm{SiO}_{2}$ substrates and indented by AFM tip. This information is available free of charge via the Internet at http://pubs.acs.org.

\section{References and Notes}

(1) Looger, L. L.; Dwyer, M. A.; Smith, J. J.; Hellinga, H. W. Nature 2003, 423, 185-190. 
(2) Yasutomi, S.; Morita, T.; Imanishi, Y.; Rimura, S. Science 2004, 304, 1944-1947.

(3) Maruccio, G.; Biasco, A.; Visconti, P.; Bramanti, A.; Pompa, P. P.; Calabi, F.; Cingolani, R.; Rinaldi, R.; Corni, S.; Di Felice, R.; Molinari, E.; Verbeet, M. P.; Canters, G. W. Adv. Mater. 2005, 17, 816-822.

(4) Graveland-Bikker, J. F.; Schaap, I. A. T.; Schmidt, C. F.; de Kruif, C. G. Nano Lett. 2006, 4, 616-621.

(5) Rochet, J. C., Jr; Lansbury, P. T. Curr. Opin. Struct. Biol. 2000, 10, 60-68.

(6) Dobson, C. M. Philos. Trans. R. Soc. London, Ser. B 2001, 356, 133 145.

(7) Perutz, M. F.; Finch, J. T.; Berriman, J.; Lesk, A. Proc. Natl. Acad. Sci. U.S.A. 2002, 99, 5591-5595.

(8) Makin, O. S.; Atkins, E.; Sikorski, P.; Johansson, J.; Serpell, L. C. Proc. Natl. Acad. Sci. U.S.A. 2005, 102, 315-320.

(9) Pepys, M. B. Philos. Trans. R. Soc. London, Ser. B. 2001, 356, 203 210.

(10) Reches, M.; Gazit, E. Science 2003, 300, 625-627.

(11) Scheibel, T.; Parthasarathy, R.; Sawicki, G.; Lin, X. M.; Jaeger, H.; Lindquist, S. L. Proc. Natl. Acad. Sci. U.S.A. 2003, 100, 4527-4532.

(12) Zhang, S. Nat. Biotechnol. 2003, 21, 1171-1178.

(13) Kasai, S.; Ohga, Y.; Mochizuki, M.; Nishi, N.; Kadoya, Y.; Nomizu, M. Biopolymers 2004, 76, 27-33.

(14) Horii, A.; Wang, X.; Gelain, F.; Zhang, S PLoS ONE 2007, 2, e190.

(15) Guo, S.; Akhremitchev, B. B. Biomacromolecules 2006, 7, 1630-1636

(16) Smith, J. F.; Knowles, T. P.; Dobson, C. M.; MacPhee, C. E.; Welland, M. E Proc. Natl. Acad. Sci. U.S.A. 2006, 103, 15806-15811.

(17) Kol, N.; Adler-Abramovich, L.; Barlam, D.; Shneck, R. Z.; Gazit, E.; Rousso, I Nano Lett. 2005, 5, 1343-1346.

(18) Hertz, H. J. Reine Angew. Math. 1881, 92, 156-171.

(19) Boussinesq, J. Application des potentiels al'etude de l'equilibre et du mouvement des solides elastiques; Gauthier-Villars: Paris 1885.

(20) Sneddon, I. N. Int. J. Eng. Sci. 1965, 3, 47-57.

(21) Flamia, R.; Zhdan, P. A.; Martino, M.; Castle, J. E.; Tamburro, A. M. Biomacromolecules 2004, 5, 1511-1518.

(22) Flamia, R.; Salvi, A. M.; D’Alessio, L.; Castle, J. E.; Tamburro, A. M Biomacromolecules 2007, 8, 128-38.

(23) Debelle, L.; Tamburro, A. M Int. J. Biochem. Cell Biol. 1999, 31 261-272.

(24) Varadi, D. P. J. Invest. Dermatol. 1972, 59, 238-246.

(25) Uitto, J.; Paul, J. L.; Brockley, K.; Pearce, R. H.; Clark, J. G. Lab. Invest. 1983, 49, 499-505.

(26) Tamburro, A. M.; Bochicchio, B.; Pepe, A. Biochemistry 2003, 42, 13347-13362.

(27) Tamburro, A. M.; De Stradis, A.; D’Alessio, L. J. Biomol. Struct. Dyn. 1995, 12, 1161-1172.

(28) Tamburro, A. M.; Daga Gordini, D.; Guantieri, V.; De Stradis, A. In Chemistry and Properties of Biomolecular Systems; Russo, N., Anastassopoulou, S., Barone, G., Eds.; Kluwer Publishers: Dordrecth, 1994; Vol. 2, pp 389-403..

(29) Tamburro, A. M.; Guantieri, V. In Ecological Physical Chemistry; Rossi, C., Tiezzi E, Eds.; Elsevier: Amsterdam, 1991; Vol. 391.
(30) Castiglione Morelli, M. A.; De Biasi, M.; De Stradis, A.; Tamburro, A. M. J. Biomol. Struct. Dyn. 1993, 11, 181-190.

(31) Bochicchio, B.; Floquet, N.; Pepe, A.; Alix, A. J. P.; Tamburro, A. M Chemistry 2004, 10, 3166-3176.

(32) Tamburro, A. M.; Guantieri, V.; Daga-Gordini, D. J. Biomol. Struct. Dyn. 1992, 10, 441-454.

(33) Rodríguez-Cabello, J. C.; Prieto, S.; Reguera, J.; Arias, F. J.; Ribeiro, A. J. Biomater. Sci., Polym. Ed. 2007, 18, 269-286.

(34) Martino, M.; Perri, T.; Tamburro, A. M Macromol. Biosci. 2002, 2, 319-328.

(35) Burnham, N. A.; Colton, R. J. J. Vac. Sci. Technol. 1989, 71, 29062913.

(36) Moriarty, D. F.; Raleigh, D. P. Biochemistry 1999, 38, 1811-1818.

(37) Nilsson, M. R. Methods 2004, 34, 151-160.

(38) Lefèvre., T.; Arsenault, K.; Pezolet, M. Biopolymers 2004, 73, 705715

(39) Susi, H.; Timasheff, S. N.; Stevens, L. J. J. Biol. Chem. 1967, 242, 5467-5473.

(40) Clark, A. H.; Saunderson, D. H. P.; Suggett, A Int. J. Pept. Protein Res. 1981, 17, 353-364.

(41) Bandekar, J. Biochim. Biophys. Acta 1992, 1120, 123-143.

(42) Andries, J. C.; Anderson, J. M.; Walton, A. G. Biopolymers 1971, 10, 1049-1057.

(43) Klunk, W. E.; Pettegrew, J. W.; Abraham, D. J. J. Histochem. Cytochem. 1989, 37, 1273-81.

(44) LeVine, III H Amyloid 1995, 2, 1-6.

(45) Naiki, H.; Higuchi, K.; Hosokawa, M.; Takeda, T. Anal. Biochem. 1989, 177, 244-249.

(46) Luckey, M.; Hernandez, J. F.; Arlaud, G.; Forsyth, T.; Ruigrok, R. W. H.; Mitraki, A. FEBS Lett. 2000, 468, 23-27.

(47) Jansen, R.; Dzwolak, W.; Winter, R. Biophys. J. 2005, 88, 13441353.

(48) Jimenez, J. L.; Nettleton, E. J.; Bouchard, M.; Robinson, C. V.; Dobson, C. M.; Saibil, H. R. Proc. Natl. Acad. Sci. U.S.A. 2002, 14, 9196-201.

(49) Johnson, K. L. Contact mechanics; Cambridge University Press: Cambridge, U.K. 1985.

(50) Akhremitchev, B. B.; Walker, G. C. Langmuir 1999, 15, 5630-5634.

(51) Dimitriadis, E. K.; Horkay, F.; Maresca, J.; Kachar, B.; Chadwick, R. S. Biophys. J. 2002, 82, 2798-2810.

(52) Aaron, B. B.; Gosline, J. M. Biopolymers 1981, 20, 1247-1260.

(53) Martino, M.; Perri, T.; Tamburro, A. M. Biomacromolecules 2002, 3, 297-304.

(54) Tamburro, A. M.; Pepe, A.; Bochicchio, B.; Pasquali Ronchetti, I. J. Biol. Chem. 2005, 280, 2682-2690.

(55) Guantieri, V.; Tamburro, A. M.; Daga-Gordini, D. Connect. Tissue Res. 1983, 12, 79-83.

(56) Harris, J. R. Micron 2002, 33, 609-626.

(57) Fan, K.; Nagle, W. A. BMC Pulm. Med. 2002, 2, 1-5.

(58) Faury, G. Pathol. Biol. 2001, 49, 310-325.

(59) Fung, Y. C. Biomechanics. Mechanical Properties of Living Tissues; Springer-Verlag: New York, 1993.

BM7010104 